\title{
The Guillain-Barrè peptide signatures: from Zika virus to Campylobacter, and beyond
}

\author{
This article was published in the following Dove Press journal: \\ Virus Adaptation and Treatment \\ I August 2017 \\ Number of times this article has been viewed
}

\author{
Guglielmo Lucchese' \\ Darja Kanduc ${ }^{2}$ \\ 'Brain Language Laboratory, \\ Freie Universität Berlin, Berlin, \\ Germany; ${ }^{2}$ Department of \\ Biosciences, Biotechnologies and \\ Biopharmaceutics, University of Bari, \\ Bari, Italy
}

Abstract: Scientific attention has focused recently on the link between Guillain-Barrè syndrome (GBS) and Zika virus (ZIKV). Two related questions emerged: 1) what triggered the violent 2014 outbreak of a virus, which, first identified in 1947, had caused only a limited number of documented cases of human infection until 2007 and 2) which molecular mechanism(s) relate ZIKV active infection to GBS, an autoimmune inflammatory polyradiculoneuropathy. Capitalizing on the increased interest on ZIKV and hypothesizing the involvement of autoimmune mechanisms, we searched for minimal epitopic determinants shared between ZIKV and other GBS-related pathogens - namely, Epstein-Barr virus, human cytomegalovirus, influenza virus, Campylobacter jejuni, and Mycoplasma pneumoniae, among others - and human proteins that, when altered, have been associated with myelin disorders and axonopathies. We report a considerable peptide matching that links GBS-related pathogens to human proteins related to myelin disorders and axonopathies. Crucially, the shared pentapeptides repeatedly occur throughout numerous epitopes validated as immunopositive by a conspicuous scientific literature. The data support a scenario where multiple different infections over time and resulting multiple cross-reactions may contribute to the pathogenesis of GBS. In practice, previous infection(s) might create immunologic memory able to trigger uncontrolled hyperimmunogenicity during a successive pathogen exposure. ZIKV pandemic appears to be an exemplar model for a proofof-concept of such multiple cross-reactivity mechanism.

Keywords: peptide sharing, GBS-related human proteins, GBS-related pathogens, multiple cross-reactivity, hyperimmunogenicity

\section{Introduction}

Zika virus (ZIKV) was discovered in 1947 and has been considered of little or no clinical importance until 2007, a date that marks the beginning of epidemics that caught scientific and clinical communities by surprise. ${ }^{1}$ Among the pathologic sequelae, Guillain-Barré syndrome (GBS) in adults appears to be a clinical outcome following ZIKV active infection. ${ }^{2-7}$

How this neuropathologic outcome and the flavivirus infection may be linked at the molecular level is unknown. Actually, some authors have even interpreted the co-occurrence of ZIKV infection and GBS as a temporal coincidence rather than a causal relation. ${ }^{8}$ The issue is further complicated by the fact that GBS - although described for the first time in $1859^{9}$ - still presents, beyond its association with ZIKV, a largely unknown etiology. Genetic factors have been hypothesized to constitute a causal platform leading to the disease. ${ }^{10,11}$ However, research on candidate genes that could plausibly be involved in the pathogenesis of GBS has not been conclusive. For
Correspondence: Darja Kanduc Department of Biosciences, Biotechnologies and Biopharmaceutics, University of Bari, Via Orabona 4, Bari 70126, Italy

Tel +390805443 321

Fax +390805443 317

Email dkanduc@gmail.com 
instance, increased susceptibility to GBS in association with polymorphisms in CD1, low-affinity immunoglobulin gamma Fc region receptors II-a and II-b (FcRII-a and FcRII-b), ${ }^{12-14}$ and interleukin-10 genes still awaits validation. ${ }^{15-17}$

In parallel, a large body of research has investigated the possible association between GBS and infections. In particular, Campylobacter jejuni and Mycoplasma pneumoniae infections have been related to the disease, ${ }^{18-22}$ most possibly through autoimmune cross-reactive mechanisms. ${ }^{23-26}$ At a lesser extent, human cytomegalovirus (HCMV) and Epstein-Barr virus (EBV) might also be involved in GBS. Indeed, although many reports did not relate HCMV to GBS, ${ }^{27-29}$ nonetheless, numerous cases of HCMV-GBS association have been described in transplant recipients and during pregnancy. ${ }^{30-36}$ Moreover, a prospective cohort study described $63(12.4 \%)$ HCMV-GBS cases out of 506 patients with cases of GBS, with patients with HCMV-GBS more likely to be young $-<35$ years old in most of the cases - and female. ${ }^{37}$ Similarly, numerous clinical case reports describe GBS associated with EBV infection. ${ }^{38-42}$

However, it remains unclear how such infectious pathogens - which are largely widespread and sluggishly latent worldwide ${ }^{43-48}$ - can, all of a sudden, attack the host causing the complex pathologic picture of the GBS.

Based on the autoimmune context that connects infections to GBS, we recently analyzed ZIKV polyprotein for peptide sharing with human proteins that, when altered, may associate with GBS-like syndromes and found large peptide overlap suggestive of a great potential for autoimmune crossreactivity. ${ }^{49}$ Here, we use the peptide platform common to ZIKV and GBS-associated human proteins and search for minimal immune determinants that are additionally shared with infections related to GBS such as the above-mentioned C. jejuni, HCMV, EBV, and M. pneumoniae. The scientific rationale is that different infectious pathogens might evoke a succession of immune responses converging on identical epitopic sequences and characterized by a progressively more rapid production of increasingly powerful antibodies on subsequent encounters with the same epitopic targets. Then, sharing of identical cross-reactive epitopes with host proteins can result in amplified cross-reactions and consequent severe diseases, thus explaining the violence of the otherwise asymptomatic ZIKV infection. ${ }^{50}$

\section{Methods}

Analyses were conducted on human proteins related to GBS and retrieved from UniProtKB Database (http://www.uniprot. org/) $)^{51}$ using "myelin, (de)myelination, axonal neuropathy" as keywords (Table S1), as already detailed elsewhere. ${ }^{50}$ References for disease involvement are available at http://www. uniprot.org/. Human proteins are indicated by the UniProt Accession name.

A set of pathogen proteomes was chosen for analyses based on the following criteria:

- belonging to infectious agents that have been reported as related to or concomitant, even occasionally, with GBS, ie, C. jejuni, ${ }^{18,19}$ M. pneumoniae, ${ }^{18,20-22} \mathrm{HCMV},{ }^{18,30-37}$ EBV, ${ }^{18,38-42}$ hepatitis E virus, genotype 1 (HEV), ${ }^{52}$ human papillomavirus type 16 (HPV16), ${ }^{53}$ influenza viruses, ${ }^{54}$ dengue virus (DENV), ${ }^{55}$ West Nile virus (WNV), ${ }^{56}$ yellow fever virus (YFV), ${ }^{57}$ and varicella zoster virus (VZV) ${ }^{58}$

- for which proteome completeness has been established;

- with experimental evidence at protein level;

- belonging to the Swiss-Prot section reviewed by UniProtKB.

The pathogen proteomes are as follows (in alphabetical order, with abbreviations, number of proteins, number of amino acids (aa), and taxonomy ID): C. jejuni, 1623 proteins, 507643 aa (192222); DENV, 10 proteins, 3392 aa (11059); EBV, 109 proteins, 51458 aa (10377); HEV, 3 proteins, 2467 aa (652674); HCMV, strain Merlin, 168 proteins, 63460 aa (295027); HPV16, 8 proteins, 2426 aa (333760); influenza A virus, H1N1, 12 proteins, 4788 aa (211044); influenza A virus, H5N5, 12 proteins, 4809 aa (93838); influenza B virus, 11 proteins, 4718 aa (518987); M. pneumoniae, 687 proteins, 239888 aa (272634); VZV, 69 proteins, 35782 aa (10338); WNV, 13 proteins, 3430 aa (11082); YFV 13 proteins, 3411 aa (11090); and ZIKV, 13 proteins, 3419 aa (64320). Proteomes are described in detail at http://www.uniprot.org/. ${ }^{51}$

Peptide matching analyses were conducted using the pentapeptide as a minimal immune unit ${ }^{59-61}$ and utilizing the elsewhere described pentapeptide platform shared between ZIKV and human proteins that, when altered, may associate with GBS. ${ }^{49}$ In brief, GBS-related proteins were obtained from UniProtKB ${ }^{51}$ using the keywords "myelin, (de)myelination, axonal neuropathy". Then, the primary aa sequence of each ZIKV protein was dissected into pentapeptides overlapping each other by four residues. For example, ZIKV protein C (Q32ZE1, aa 2-104, KNPKEEIRRIRIVNMLKRGVARVNPLGGLKRLPAGLLLGHGPIRMVLAILAFLRFTAIKPSLGLINRWGSVGKKEAMEIIKKFKKDLAAMLRIINARKERKRR) was sequentially dissected into KNPKE, NPKEE, PKEEI, KEEIR, and so forth until its last pentapeptide ERKRR, for a total of 99 pentamers. At the end, the $12 \mathrm{ZIKV}$ proteins 
(prM considered as one protein) yielded 3370 pentapeptides. The same procedure was applied to calculate the number of pentapeptides present in the pathogen proteomes described earlier.

The 3370 ZIKV pentapeptides were probed for occurrences within the set of human proteins related to GBS using PIR peptide match program (http://research.bioinformatics. udel.edu/peptidematch/index.jsp). ${ }^{62}$ A total of $222 \mathrm{ZIKV}$ pentapeptides were found to occur throughout 97 human proteins related to GBS. The 97 human proteins related to GBS and sharing pentapeptide(s) with ZIKV have been previously detailed. ${ }^{49}$

The pentapeptide platform common to ZIKV and GBSrelated proteins was used to search for commonalities with GBS-related pathogens. That is, each of the 222 ZIKV pentapeptides shared with the 97 GBS-related proteins was analyzed for occurrences in the pathogen proteomes of infectious agents that had been selected as described earlier.

The immunological potential of the peptide sharing was investigated using the Immune Epitope Database (IEDB; www.iedb.org) resource. ${ }^{63}$ Only epitopes that had been experimentally validated as immunopositive in the human host were considered.

\section{Results}

\section{Pentapeptide(s) common to ZIKV, GBS-related human proteins, and GBS-related pathogens.}

Table 1 shows the occurrences of the ZIKV pentapeptides shared with the 97 GBS-related proteins in the analyzed infectious agents. It can be seen that, with the exception of influenza B virus, the analyzed GBS-associated pathogens share pentapeptides common to ZIKV and human GBSrelated proteins.

Numerically, 135 out of 222 pentapeptides shared between ZIKV and the set of human GBS-related proteins occur and often recur throughout the pathogens under analysis for a total of 206 multiple occurrences. As previously observed, ${ }^{64-67}$ such pentapeptide sharing is extremely high

Table I Pentapeptides common to ZIKV, GBS-related human proteins, and GBS-related pathogens

\begin{tabular}{|c|c|}
\hline Pentapeptides $^{\mathbf{a}}$ & Pathogen \\
\hline- & Influenza B virus \\
\hline EEIRR, PTQGS & Influenza $\mathrm{A}$ virus $(\mathrm{H} 5 \mathrm{NI})$ \\
\hline ASSLV, EEIRR & Influenza $\mathrm{A}$ virus $(\mathrm{HINI})$ \\
\hline TLETI, TVEVQ & HPVI6 \\
\hline AAARA, ALRGL, LAAAV, LRGLP, RLAAA & HEV, genotype $I^{b}$ \\
\hline AEEVL, ALAGA, ALAGG, GERAR, LAGAL, LLSLK, LLVVL, SPGAG, TAVSA, VLTAV & EBV \\
\hline EALIT, FATTL, GAALR, GAGKT, GALEA, IFLST, ILAAL, LLLGR, LLLLT, LRIIN, RRLLG, TVSLG, VLTAV & VZV \\
\hline $\begin{array}{l}\text { AAARA, EEEKE, EEIRR, FDLEN, GAALG, GAGKT, GEAAA, GTVSL, ILAAL, LLALA, LLGLL, LLLGR, LQDGL, } \\
\text { LTAVR, LTCLA, LWLLR, SLGLD, STSQK, TAVSA, TVSLG }\end{array}$ & HCMV \\
\hline $\begin{array}{l}\text { AAAIF, AARGY, CSAVP, DRRWC, EFEAL, EFGKA, ETLGE, GCGLF, GDTAW, GEAAA, GETLG, GPSLR, } \\
\text { GRARV, GSASS, GVPLL, LNDMG, NSTHE, QRGSG, RDLRL, RGYIS, RRDLR, RRWCF, RVILA, SAVPV, } \\
\text { TAAGI, YISTR }\end{array}$ & DENV \\
\hline $\begin{array}{l}\text { AAAIF, AALGA, AARGY, ALAGA, ALGAI, ALRGL, ALVAV, ASAGI, ASSLV, CSAVP, DENHP, DRRWC, } \\
\text { EALRG, EFEAL, EFGKA, ESSSS, FATTL, GAGKT, GCGLF, GDTAW, GEAAA, GRARV, GSASS, KGIGK, } \\
\text { LAAAV, LRGLP, LVNGV, NSTHE, PRRLA, QRGSG, RDLRL, RGYIS, RRDLR, RRWCF, SAVPV, SLFGG, } \\
\text { SPGAG, TAAGI, TEVEV, TKEEF, VEGLG, VSRGS, VTLGA, YISTR }\end{array}$ & WNV \\
\hline EFEAL, EFGKA, ESSSS, GAGKT, GCGLF, GDTAW, LKDGR, NSTHE, QRGSG, TKEEF & YFV \\
\hline $\begin{array}{l}\text { AAAIF, AAARA, ALAGG, ALEAE, ALGAI, ALGLT, DGLSE, EEARR, EGLKK, ENEAL, ESSSS, EVEET, GAGKT, } \\
\text { GALEA, GLKKR, GPSLR, GSASS, IILLV, ILAAL, ILLMV, ISALE, KEVKK, LAAAV, LAGAL, LKDGR, LKGKG, } \\
\text { LLAVP, LLGLL, LLLLT, LLTTA, LLVVL, LQDGL, LVEED, LVILL, NGVQL, PTQGS, RLAAA, RRALK, SLGLD, } \\
\text { TEVEV, TKEEF, VEFKD, VVDPI, VVGLL }\end{array}$ & M. pneumoniae \\
\hline $\begin{array}{l}\text { AALGA, AKVEV, ALAGA, ALAGG, ALGAI, ALGLT, ALKDG, ALVAV, ASAGI, ASSLV, DENHP, DGLSE, } \\
\text { EALIT, EALRG, EEEKE, EELEI, EFEAL, EGLKK, EKEWK, ENEAL, ENIKD, FDLEN, GAALG, GAGKT, GALEA, } \\
\text { GEAGA, GETLG, GGGTG, GIMLL, GPSLR, GSASS, GTLPG, IFLST, IILLV, ILAAL, ILLMV, KEVKK, KGIGK, KGSLV, } \\
\text { KKSGI, KNPKE, LAGAL, LALGG, LALGG, LDFSD, LKGKG, LLALA, LLAVP, LLGLL, LLLGR, LLLLT, LLSLK, } \\
\text { LLVVL, LRIIN, LSTQV, LTAVG, LVILL, LVNGV, MLLSL, PRRLA, PSLGL, RRALK, RVILA, SEELE, SLFGG, SLGLI, } \\
\text { STSQK, STTAS, TAAGI, TEVEV, TKEEF, TLETI, VEEDG, VEFKD, VEGLG, VLSMV, VNPLG, VRAAK, VSRGS, } \\
\text { VTLGA, VVGLL, YLSTQ }\end{array}$ & C. jejuni \\
\hline
\end{tabular}

Notes: aPentapeptides with multiple occurrences in bold. ' $T$ wo HEVs, genotype 3, taxonomy IDs 5096/5 and 5/2345, share only 2 pentapeptides (ALRGL and LRGLP). Abbreviations: ZIKV, Zika virus; GBS, Guillain-Barrè syndrome; HPVI6, human papillomavirus type 16; HEV, hepatitis E virus, genotype I; EBV, Epstein-Barr virus; VZV, varicella zoster virus; HCMV, human cytomegalovirus; DENV, dengue virus; WNV, West Nile virus; YFV, yellow fever virus. 
and unexpected given that the probability E of a ZIKV pentapeptide to occur simultaneously in the set of the 13 GBSrelated pathogens and in the set of the 97 human GBS-related proteins is $6977141836772461 \mathrm{e}-6$, ie, it is close to zero (Box 1). This infinitesimally low value is in sharp contrast with the actual data displayed in Table 2. As a matter of fact, Table 2 highlights an intense pentapeptide sharing that underlies a multiple cross-reactivity platform among the analyzed pathogens and the human host.

\section{Immune potential of the pentapeptides common to ZIKV, GBS-related human proteins, and GBS-related pathogens}

Such a high potential for cross-reactions appears likely also in view of the fact that most of the 135 pentapeptides detailed in Table 2 not only often recur among the GBSrelated pathogens (eg, the pentapeptides EEIRR, AAARA, ALAGG, GAGKT, GALEA, GPSLR, and GSASS given in bold in Table 1) but are also present in hundreds of epitopes experimentally validated as immunopositive in the human host. Table 2 shows a limited representative list of such immunopositive epitopic sequences.

\section{Discussion}

Starting from $2000,{ }^{64}$ our laboratory described a massive peptide overlap between proteins from infectious agents and the human proteome ${ }^{65}$ thus calling attention to the cross-reactivity issue in immunology. In fact, the magnitude of such a peptide sharing leads to predict a high extent of cross-reactive immune responses following infections in the human host. ${ }^{66-72}$

Here, we focus on the issue of multiple cross-reactivity and analyze the molecular connections between infectious pathogens related to GBS. We report on the presence of minimal immune determinants in the human host and repeatedly shared among infectious agents so different as the flaviviruses ZIKV, DENV, WNV, and YFV and the bacteria M. pneumoniae and C. jejuni. Such intrapathogen peptide commonality may originate multiple cross-reactions having the same peptide sequences as epitopic targets. The immunological implications are that immune responses elicited by different successive infections may add up with intensified avidity and affinity at the level of cross-reactive sites, thus exacerbating autoimmune attacks in the host.

The extent of the autoimmune damage emerges from the analysis of the human proteins involved in the sharing, most of which are crucial components of the neurological network. An example among the many is the pentapeptide LAGAL that is shared by ZIKV, EBV, M. pneumoniae, and $C$. jejuni and is also present in three human proteins involved in myelin disorders, ie, CGT, GFAP, and MTMR2 (Tables S1 and S2).

- CGT or 2-hydroxyacylsphingosine 1-beta-galactosyltransferase is involved in the synthesis of sulfatide 3-O-sulfogalactosylceramide, ${ }^{73}$ which is essential for paranodal junction formation and for the maintenance of ion channels on myelinated axons. ${ }^{74}$ Of note, the sulfatide blocks the binding of $C$. jejuni DNA-binding protein to

Box I Theoretical probability E of a ZIKV pentapeptide to occur simultaneously in the set of the I3 GBS-related pathogens and in the set of the 97 human GBS-related proteins under analysis

The expected number of times $E$ that one pentapeptide occurs in a protein is directly proportional to the number $p$ of pentapeptides in the protein and inversely related to the number $\mathrm{N}$ of all possible pentapeptides, with $\mathrm{N}$ equal to $20^{5}$ since each pentapeptide residue can be any of 20 aa and assuming that all aa occur with the same frequency: accordingly, such expected number of times $\mathrm{E}$ is given by equation

$E=\frac{P}{N}$

By considering two proteins, I and 2, of pentapeptide size $\mathrm{pI}$ and $\mathrm{p} 2$, and two events $\mathrm{EI}$ and E2 - that are assumed to be independent - that a same pentapeptide will be selected simultaneously in both proteins $I$ and 2 , then the expected number of times $E$ is given by

$\mathrm{E}=\mathrm{El} \bullet \mathrm{E} 2=\frac{\mathrm{P}_{1}}{\mathrm{~N}} \bullet \frac{\mathrm{P}_{2}}{\mathrm{~N}}=\frac{\mathrm{P}_{1} \bullet \mathrm{P}_{2}}{\mathrm{~N}^{2}}$

In the case in point, the expected number of times $E$ that a ZIKV pentapeptide will be simultaneously present in the set formed by the 97 human GBS-related proteins (Table SI) and in the set of the I3 GBS-related pathogen proteins is given by the formula:

$\mathrm{E}=\frac{P_{\text {ZIKV }} \bullet P_{\text {human }} \bullet P_{\text {pathogens }}}{\mathrm{N}^{3}}$

where $P_{\text {ZIKV }}=3370$ (no of ZIKV pentapeptides), $P_{\text {human }}=74004$ (no of pentapeptides contained in the set of 97 human GBS-related proteins), $\mathrm{P}_{\text {pathogens }}=916732$ (no of pentapeptides contained in the set of proteins from the 13 GBS-related pathogens), and $\mathrm{N}^{3}=20^{15}$ (with $\mathrm{N}$ equal to $20^{5}$ ). Solving the equation, one obtains $E=6,977|4| 83677246$ le-6.

Abbreviations: ZIKV, Zika virus; GBS, Guillain-Barrè syndrome; aa, amino acids. 
Table 2 Epitopes experimentally validated as immunopositive in the human host and containing pentapeptide(s) common to ZIKV, GBS-related human proteins, and GBS-related pathogens

\begin{tabular}{|c|c|c|c|}
\hline $\begin{array}{l}\text { IEDB } \\
\text { ID }^{\mathrm{a}}\end{array}$ & Epitope $^{\mathrm{b}, \mathrm{c}}$ & $\begin{array}{l}\text { IEDB } \\
\text { ID }^{\mathbf{a}}\end{array}$ & Epitope ${ }^{b, c}$ \\
\hline 10 & aaAAAIFvi & 446920 & nIISALEea \\
\hline 2859 & alRGLPiry & 452231 & ALAGGitmv \\
\hline 3546 & apfdETLGEedkdld & 452923 & aseALAGAL \\
\hline 9980 & dRGYISqy & 454329 & gSLGLIfal \\
\hline 11125 & EALRGLPir & 454747 & illdhEKEWKI \\
\hline 14278 & ESSSSdkp & 455521 & kLQDGLlhi \\
\hline 15968 & fGDSYI & 456221 & LLAVPvpgv \\
\hline 19121 & GDSYli & 456552 & IprgLAGAL \\
\hline $2|27|$ & gmGEAAAIF & 458233 & rlvGIMLLI \\
\hline 21783 & GPSLRtttv & 459912 & tIDENHPsi \\
\hline 24302 & hlsLRGLPv & 460498 & vpAAARAgaia \\
\hline 36077 & IGDTAW & 462972 & ALAGApyqa \\
\hline 37757 & IIrSTSQK & 464145 & dtyPALLVv \\
\hline 42819 & mTKEEFtry & 465337 & ftASAGlqv \\
\hline 43963 & nGCGLF & 465643 & GLDFSIpgm \\
\hline 47494 & PFGDSy & 467239 & kLEGDLtgpsv \\
\hline 59141 & slgLVILLvl & 469571 & qpEGLKKtl \\
\hline 59561 & sLVNGVvrl & 470656 & sLLTTAevvr \\
\hline 62564 & syhDRRWCF & 471899 & tpfGGGTGgf \\
\hline 64181 & tiaydEEARR & 472107 & TVSLGgfeitppv \\
\hline 71730 & vvlaGAALGvataaq & $4754 I I$ & AETDEprll \\
\hline 76146 & ytpgsclagvIEALIThqre & 475437 & aeVEGLGkgva \\
\hline 95619 & ngttrtVNPLGf & 475912 & apSSTSQel \\
\hline 108957 & fLLGLLffv & 477278 & EEEKErntaa \\
\hline 113368 & elilydkEEIRRi & 478632 & gidSSSPEv \\
\hline|| $852 \mid$ & mILKGKGdkaqie & 479191 & hENEALwreva \\
\hline 133702 & srNSTHEmy & 480858 & klrEEARRk \\
\hline 162369 & gQRGSGssf & 481247 & KTKDGvrev \\
\hline 162647 & kEVEETata & 483586 & qSTTASIskk \\
\hline 162892 & IpktGTVSL & 483862 & rEFGKAlql \\
\hline 176418 & rALEAEkralw & 484166 & RLAAAarek \\
\hline 179238 & maflrsvsRLAAAVf & 485074 & ryiEGLKKR \\
\hline 179920 & vvRDLRLra & 485864 & sfSPGAGaf \\
\hline 180696 & pasiAARGYi & 486103 & SLFGGtsgl \\
\hline 180816 & wgnGCGLFgkggivt & $486 \mid 46$ & sLLALAGAv \\
\hline 182553 & allaLNDMGk & 487020 & sVSRGSslk \\
\hline |85931 & tkqtGSASSm & 487072 & sygpGPSLR \\
\hline 188820 & avgtgtGAALGAgigALAGG & 487689 & trhKEVKKI \\
\hline $18892 \mid$ & mntkiatrIsvfALAGAlla & 488045 & VEEDGqlksl \\
\hline 19440I & tLAAAVpki & 488605 & vVVDPIIsk \\
\hline 195482 & iGETLGEkwksrlna & 491301 & GRARVsvev \\
\hline 207331 & ELGKRvqal & 492165 & hrALVAVII \\
\hline 209443 & gesGAGKTw & 493078 & IrfpNGVQL \\
\hline 213577 & ktfTAAGI & 494432 & rrILLLLTI \\
\hline 217605 & REEGAvdksy & 506799 & LLTRScail \\
\hline 220715 & vEVQLLeskty & 506908 & IPRRLAiql \\
\hline 220742 & vGAALRpaf & 510341 & ypASSLVv \\
\hline 222394 & GEAGAiervl & 515064 & epGEAAAggaaEEARR \\
\hline 222457 & geGLLIVkv & 536076 & GTLPGsaeppLTAVR \\
\hline 222756 & heySEELEkl & 539058 & ahftdpssvAARGYISt \\
\hline 222989 & kegyVLTAV & 539081 & ALRGLPvry \\
\hline 236364 & VRAAKfwk & 539140 & CSAVPVdw \\
\hline 420040 & qnpqILAAL & 539287 & GEAAAIFmt \\
\hline 420274 & tPTQGSvl & 539600 & ImyfhRRDLRLasna \\
\hline
\end{tabular}

(Continued)
Table 2 (Continued)

\begin{tabular}{llll}
\hline $\begin{array}{l}\text { IEDB } \\
\text { ID }^{\mathbf{a}}\end{array}$ & Epitope $^{\mathrm{b}, \mathrm{c}}$ & $\begin{array}{l}\text { IEDB } \\
\text { ID }^{\mathbf{a}}\end{array}$ & Epitope $^{\mathrm{b}, \mathrm{c}}$ \\
\hline 423402 & IFATTLfigympihc & 541359 & dAALGAeem \\
423807 & aaaVVGLy & 541798 & eLLSLKy \\
424246 & EEARRLLGy & 542210 & GAALRGLsI \\
427527 & stTAVSAry & $54231 \mathrm{I}$ & ghtLLALAt \\
427966 & twnAVLLRy & 542373 & gLKDGVall \\
$43574 I$ & sRVILAgnll & 544887 & RRLLGkykf \\
436632 & aTLETIIrh & 546136 & vLRIINeptaaai \\
437505 & GEAAAkeew & 548062 & elkLRGLPvsgt \\
\hline
\end{tabular}

Notes: ${ }^{2}$ Epitope IEDB IDs are listed in ascending numerical order. Details and references are available at http://www.iedb.org/idsearch.php. 'Epitope peptide sequences are given in one letter code. Shared peptide fragments are given in capital letters.

Abbreviations: ZIKV, Zika virus; GBS, Guillain-Barrè syndrome; IEDB, Immune Epitope Database.

myelinated nerves, a reaction that has been associated with $C$. jejuni-related GBS ${ }^{75}$

- GFAP or glial fibrillary acidic protein has been reported to be a marker of axonal GBS and outcome; ${ }^{76}$

- Myotubularin-related protein 2 (MTMR2) is a phosphoinositide-3-phosphatase that, if altered, associates with demyelinating peripheral neuropathy characterized by excessive redundant myelin, also known as myelin outfoldings. ${ }^{77,78}$ MTMR2 appears to negatively regulate membrane homeostasis in Schwann cell myelination. ${ }^{79}$

Another myotubularin-related protein, namely MTMR5, shares eight pentapeptides with ZIKV (eg, AVLLR, GPSLR, GLLIV, LQDGL, REEGA, SEELE, SLGLI, and VLSMV) and many of the said pentapeptides are also present in infectious agents (Table 1). MTMR5 pentapeptide sharing is noteworthy. Indeed, alterations of MTMR5 are involved in demyelinating neuropathies ${ }^{79}$ and, in addition and most interestingly, lead to impaired spermatogenesis. ${ }^{80-82}$ This datum might be a hint for widening the study of the still obscure reasons for gender differences in GBS pathogenesis. ${ }^{83}$ In this perspective, also future studies that extend analyses to non-peptidic GBS epitopes warrant attention. For example, B4GN1 or beta-1,4- $N$-acetylgalactosaminyltransferase 1 plays a role in spermatogenesis and, when altered, in male infertility; ${ }^{84}$ is involved in the biosynthesis of gangliosides ${ }^{85}$ and produces a ceramide trisaccharide ( $N$-acetyl-d-galactosaminyl( $N$-acetylneuraminyl)-d-galactosyl-d-glucosylceramide) that is present in non-peptidic structural GBS epitopes (IEDB IDs: 139429 and 143251).

Although space precludes a detailed discussion of the data reported in Table 1, a final note is due with regard to WNV that hosts 44 out of the 135 pentapeptides common to ZIKV and GBS-related human proteins (Table 1). WNV infections 
may cause acute flaccid paralysis through a pathogenic mechanism that most possibly involves disrupted glutamate transporter expression in the spinal cord. ${ }^{56}$ Hence, it draws our attention the presence of three pentapeptides (namely ALRGL, GAALR, and LLGLL) that are present in the amino acid transporter SATT (or SLC1A4 or ASCT1) (Table S2). SATT is essential in brain for $d$-serine transport ${ }^{86}$ and is mostly expressed in hippocampal pyramidal and dentate granule neurons, and, in the cerebellum, Purkinje cells and their dendrites, thereby suggesting a role in pathophysiological processes that involve glutamate toxicity. ${ }^{87}$ Indeed, activation of $N$-methyl-d-aspartate receptors (NMDARs) by synaptically released l-glutamate requires occupancy of coagonist binding sites in the tetrameric receptor by either glycine or d-serine, so that altered SATT and, thereby, altered d-serine flux in the brain would alter NMDAR activity. The hypothesis of a potential link between SATT-induced NMDAR alteration and flaccid paralysis seems to find a support in the fact that flaccid paraplegia has been observed in patients with autoantibodies to NMDARs. ${ }^{88-90}$

\section{Conclusion}

We observe that the main caveats of the present research are the limited number of the analyzed pathogens and, in addition, the fact that, in front of the tendency of infectious pathogens to mutate, only representative taxonomy types have been analyzed. Hence, the level of intrapathogen multiple cross-reactivity might be even underestimated. A second limitation of the present study is given by the fact that it mainly analyzes peptidic epitopic sequence potentially related to GBS. As a matter of fact, the acute paralytic GBS is also characterized by autoantibodies against glycolipids and gangliosides. ${ }^{91,92}$

Given these notes of caution, the present study offers a scientific rationale and a methodology to analyze the molecular role of intrapathogen sharing in the pathologic sequelae that may be associated with multiple infections. Indeed, the high serological cross-reactivity that exists among flaviviruses (eg, ZIKV, DENV, WNV, and YFV) ${ }^{93,94}$ exemplifies the possibility that different infections occurring at different times may sum up onto a same set of epitopic determinants and result in hyperimmunogenicity, ${ }^{95,96}$ possibly resulting in neurological damage.

\section{Acknowledgments}

GL gratefully acknowledges support from the Deutscher Akademischer Austauschdienst (DAAD), the Deutsche Forschungsgemeinschaft (DFG), and the Freie Universität
Berlin, Germany. DK's studies and research have been supported by University of Bari, Italy.

\section{Disclosure}

The authors report no conflicts of interest in this work.

\section{References}

1. Slavov SN, Otaguiri KK, Kashima S, Covas DT. Overview of Zika virus (ZIKV) infection in regards to the Brazilian epidemic. Braz J Med Biol Res. 2016;49(5):e5420.

2. Solomon IH, Milner DA, Folkerth RD. Neuropathology of Zika virus infection. J Neuroinfect Dis. 2016;7(2):220.

3. Mecharles S, Herrmann C, Poullain P, et al. Acute myelitis due to ZIKV infection. Lancet. 2016;387(10026):1481

4. Parra B, Lizarazo J, Jiménez-Arango JA, et al. Guillain-Barré syndrome associated with Zika virus infection in Colombia. $N$ Engl $J$ Med. 2016;375(16):1513-1523.

5. Cao-Lormeau VM, Blake A, Mons S, et al. Guillain-Barré Syndrome outbreak associated with Zika virus infection in French Polynesia: a case-control study. Lancet. 2016;387(10027):1531-1539.

6. Oehler E, Watrin L, Larre P, et al. Zika virus infection complicated by Guillain-Barré syndrome - case report, French Polynesia, December 2013. Euro Surveill. 2014;19(9):20720.

7. Kandel N, Lamichhane J, Tangermannc RH, Rodiera GRM. Detecting Guillain-Barré syndrome caused by Zika virus using systems developed for polio surveillance. Bull World Health Organ. 2016;94(9):705-708.

8. Leis AA, Stokic DS. Zika virus and Guillain-Barre syndrome: is there sufficient evidence for causality? Front Neurol. 2016;7:170.

9. Afifi AK. The Landry-Guillain-Barré Strohl Syndrome 1859 to 1992 A historical perspective. J Family Community Med. 1994;1(1):30-34.

10. Jasti AK, Selmi C, Sarmiento-Monroy JC, Vega DA, Anaya JM, Gershwin ME. Guillain-Barré syndrome: causes, immunopathogenic mechanisms and treatment. Expert Rev Clin Immunol. 2016;12(11):1175-1189.

11. Blum S, McCombe PA. Genetics of Guillain-Barré syndrome (GBS) and chronic inflammatory demyelinating polyradiculoneuropathy (CIDP): current knowledge and future directions. J Peripher Nerv Syst. 2014;19(2):88-103.

12. Caporale CM, Papola F, Fioroni MA, et al. Susceptibility to GuillainBarré syndrome is associated to polymorphisms of genes. J Neuroimmunol. 2006;177(1-2):112-118.

13. Myhr KM, Vågnes KS, Marøy TH, Aarseth JH, Nyland HI, Vedeler CA. Interleukin-10 promoter polymorphisms in patients with Guillain-Barré syndrome. J Neuroimmunol. 2003;139(1-2):81-83.

14. van Sorge NM, van der Pol WL, Jansen MD, et al. Severity of GuillainBarré syndrome is associated with Fc gamma Receptor III polymorphisms. J Neuroimmunol. 2005;162(1-2):157-164.

15. Kuijf ML, Geleijns K, Ennaji N, van Rijs W, van Doorn PA, Jacobs BC. Susceptibility to Guillain-Barré syndrome is not associated with CD1A and CD1E gene polymorphisms. J Neuroimmunol. 2008;205(1-2):110-112.

16. Geleijns K, Emonts M, Laman JD, et al. Genetic polymorphisms of macrophage-mediators in Guillain-Barré syndrome. J Neuroimmunol. 2007;190(1-2):127-130.

17. Dourado ME Jr, Ferreira LC, Freire-Neto FP, Jeronimo SM. No association between FCGR2A and FCGR3A polymorphisms in Guillain-Barré Syndrome in a Brazilian population. J Neuroimmunol. 2016;298:160-164.

18. Hao Q, Saida T, Kuroki S, et al. Antibodies to gangliosides and galactocerebroside in patients with Guillain-Barré syndrome with preceding Campylobacter jejuni and other identified infections. J Neuroimmunol. 1998;81(1-2):116-126.

19. Zautner AE, Johann C, Strubel A, et al. Seroprevalence of campylobacteriosis and relevant post-infectious sequelae. Eur J Clin Microbiol Infect Dis. 2014;33(6):1019-1027. 
20. Meyer Sauteur PM, Huizinga R, Tio-Gillen AP, et al. Mycoplasma pneumoniae triggering the Guillain-Barré syndrome: a case-control study. Ann Neurol. 2016;80(4):566-580.

21. Kuwahara M, Samukawa M, Ikeda T, et al. Characterization of the neurological diseases associated with Mycoplasma pneumoniae infection and anti-glycolipid antibodies. J Neurol. 2017;264(3):467-475.

22. Meyer Sauteur PM, Roodbol J, Hackenberg A, et al. Severe childhood Guillain-Barré syndrome associated with Mycoplasma pneumoniae infection: a case series. J Peripher Nerv Syst. 2015;20(2):72-78.

23. Ang CW, Jacobs BC, Laman JD. The Guillain-Barré syndrome: a true case of molecular mimicry. Trends Immunol. 2004;25(2):61-66.

24. Schirmer L, Worthington V, Solloch U, et al. Higher frequencies of HLA DQB1*05:01 and anti-glycosphingolipid antibodies in a cluster of severe Guillain-Barré syndrome. J Neurol. 2016;263(10):2105-2113.

25. Nakane S, Higuchi O, Hamada Y, et al. Ganglionic acetylcholine receptor autoantibodies in patients with Guillain-Barré syndrome. J Neuroimmunol. 2016;295-296:54-59.

26. Komagamine T, Yuki N. Ganglioside mimicry as a cause of GuillainBarré syndrome. CNS Neurol Disord Drug Targets. 2006;5(4):391-400.

27. Meléndez-Vásquez C, Redford J, Choudhary PP, et al. Immunological investigation of chronic inflammatory demyelinating polyradiculoneuropathy. J Neuroimmunol. 1997;73(1-2):124-134.

28. Lunn MP, Muir P, Brown LJ, MacMahon EM, Gregson NA, Hughes RA. Cytomegalovirus is not associated with IgM anti-myelin-associated glycoprotein/sulphate-3-glucuronyl paragloboside antibody-associated neuropathy. Ann Neurol. 1999;46(2):267-270.

29. Khalili-Shirazi A, Gregson N, Gray I, Rees J, Winer J, Hughes R. Antiganglioside antibodies in Guillain-Barré syndrome after a recent cytomegalovirus infection. J Neurol Neurosurg Psychiatry. 1999;66(3):376-379.

30. Shaban E, Gohh R, Knoll BM. Late-onset cytomegalovirus infection complicated by Guillain-Barre syndrome in a kidney transplant recipient: case report and review of the literature. Infection. 2016;44(2):255-258.

31. Alhefzi M, Aycart MA, Bueno EM, et al. Guillain-Barré syndrome associated with resistant cytomegalovirus infection after face transplantation. Transpl Infect Dis. 2016;18(2):288-292.

32. Steger CM, Antretter H, Höfer D. Guillain-Barré Syndrome due to CMV reactivation after cardiac transplantation. Case Rep Cardiol. 2012;2012:506290.

33. Papasotiriou M, Papachristou E, Marangos M, et al. Cytomegalovirus polyradiculopathy of late onset in a young renal transplant recipient. Clin Nephrol. 2013;80(1):75-78.

34. Hodowanec AC, Simon DM. Late primary cytomegalovirus infection presenting with acute inflammatory demyelinating polyneuropathy in a heart transplant recipient: a case report and review of the literature. Transpl Infect Dis. 2012;14(5):E116-E120.

35. Pollak-Christian E, Lee KS. Importance of diagnostic workup of Guillain-Barré syndrome in pregnancy. BMJ Case Rep 2016;2016:bcr2016216826.

36. Lupo J, Germi R, Jean D, et al. Guillain-Barré syndrome and cytomegalovirus infection during pregnancy. J Clin Virol. 2016;79:74-76.

37. Orlikowski D, Porcher R, Sivadon-Tardy V, et al. Guillain-Barré syndrome following primary cytomegalovirus infection: a prospective cohort study. Clin Infect Dis. 2011;52(7):837-844.

38. Bitan M, Or R, Shapira MY, et al. Early-onset Guillain-Barré syndrome associated with reactivation of Epstein-Barr virus infection after nonmyeloablative stem cell transplantation. Clin Infect Dis. 2004;39(7):1076-1078.

39. Kennedy M, Apostolova M. A rare case of infectious mononucleosis complicated by Guillain-Barre syndrome. Neurol Int. 2013;5(2):20-22.

40. Rho YI. Overlapping Guillain-Barré syndrome and Bickerstaff's brainstem encephalitis associated with Epstein Barr virus. Korean J Pediatr. 2014;57(10):457-460.

41. Communal C, Filleron A, Baron-Joly S, Salet R, Tran TA. Pediatric Miller Fisher Syndrome complicating an Epstein-Barr virus infection. Pediatr Neurol. 2016;63:73-75.
42. Masajtis-Zagajewska A, Muras K, Mochecka-Thoelke A, Kurnatowska I, Nowicki M. Guillain-Barré syndrome in the course of EBV infection after kidney transplantation - a case report. Ann Transplant. 2012;17(3):133-137.

43. Rickinson AB, Kieff E. Epstein-Barr virus. In: Knipe DM, Howley PM, editors. Fields Virology. Vol. 2. Philadelphia, PA: Lippincott, Williams \& Wilkins; 2007:2655-2700.

44. Capone G, Calabrò M, Lucchese $\mathrm{G}$, et al. Peptide matching between Epstein-Barr virus and human proteins. Pathog Dis. 2013;69(3): 205-212.

45. Saraya T, Kurai D, Nakagaki K, et al. Novel aspects on the pathogenesis of Mycoplasma pneumoniae pneumoniae and therapeutic implications. Front Microbiol. 2014;5:410.

46. Poole E, Sinclair J. Sleepless latency of human cytomegalovirus. Med Microbiol Immunol. 2015;204(3):421-429.

47. Jarvis M, Nelson J. HCMV: molecular basis of persistence and latency. In: Arvin A, Campadelli-Fiume G, Mocarski E, Moore PS, et al., editors. Human Herpesviruses: Biology, Therapy, and Immunoprophylaxis. (Chap. 42). Cambridge, UK: Cambridge University Press; 2007:765-779.

48. Tu ZC, Gaudreau C, Blaser MJ. Mechanisms underlying Campylobacter fetus pathogenesis in humans: surface-layer protein variation in relapsing infections. J Infect Dis. 2005;191(12):2082-2089.

49. Lucchese G, Kanduc D. Zika virus and autoimmunity: from microcephaly to Guillain-Barré syndrome, and beyond. Autoimmun Rev. 2016;15(8):801-808.

50. Duffy MR, Chen TH, Hancock WT, et al. Zika virus outbreak on Yap Island, Federated States of Micronesia. N Engl J Med. 2009;360(24): 2536-2543.

51. Magrane M, UniProt Consortium. UniProt knowledgebase: a hub of integrated protein data. Database (Oxford). 2011;2011:bar009.

52. Lei JH, Tian Y, Luo HY, Chen Z, Peng F. Guillain-Barré syndrome following acute co-super-infection of hepatitis $\mathrm{E}$ virus and cytomegalovirus in a chronic hepatitis B virus carrier. J Med Virol. 2017;89(2) 368-372.

53. Souayah N, Michas-Martin PA, Nasar A, et al. Guillain-Barré syndrome after Gardasil vaccination: data from vaccine adverse event reporting system 2006-2009. Vaccine. 2011;29(5):886-889.

54. Alcalde-Cabero E, Almazán-Isla J, García López FJ, et al. GuillainBarré syndrome following the 2009 pandemic monovalent and seasonal trivalent influenza vaccination campaigns in Spain from 2009 to 2011: outcomes from active surveillance by a neurologist network, and records from a country-wide hospital discharge database. BMC Neurol. 2016;16:75.

55. Umapathi T, Lim CS, Ooi EE, et al. Asymptomatic dengue infection may trigger Guillain-Barré syndrome. J Peripher Nerv Syst. 2016;21(4):375-377.

56. Blakely PK, Kleinschmidt-DeMasters BK, Tyler KL, Irani DN. Disrupted glutamate transporter expression in the spinal cord with acute flaccid paralysis caused by West Nile virus infection. J Neuropathol Exp Neurol. 2009;68(10):1061-1072.

57. Vanderley Oliveira AC, da Mota LMH, dos Santos-Neto LL, et al. Occurrence of autoimmune diseases related to the vaccine against yellow fever. Autoimmune Dis. 2014;2014:473170.

58. Yoshikawa T, Suzuki K, Suga S, et al. Immune response to gangliosides in a case of Guillain-Barré syndrome after varicella. Arch Dis Child. 2000;83(2):172-173.

59. Frank A. Immunology and Evolution of Infectious Disease. Princeton, NJ: Princeton University Press; 2002.

60. Zeng W, Pagnon J, Jackson DC. The C-terminal pentapeptide of LHRH is a dominant $\mathrm{B}$ cell epitope with antigenic and biological function. $\mathrm{Mol}$ Immunol. 2007;44(15):3724-3731.

61. Kanduc D. Pentapeptides as minimal functional units in cell biology and immunology. Curr Protein Pept Sci. 2013;14(2):111-120.

62. Chen C, Li Z, Huang H, Suzek BE, Wu CH, UniProt Consortium. A fast Peptide Match service for UniProt knowledgebase. Bioinformatics. 2013;29(21):2808-2809 
63. Vita R, Overton JA, Greenbaum JA, et al. The immune epitope database (IEDB) 3.0. Nucleic Acids Res. 2015;43(Database issue):D405-D412.

64. Natale C, Giannini T, Lucchese A, Kanduc D. Computer-assisted analysis of molecular mimicry between human papillomavirus 16 E7 oncoprotein and human protein sequences. Immunol Cell Biol. 2000;78(6):580-585.

65. Kanduc D, Stufano A, Lucchese G, Kusalik A. Massive peptide sharing between viral and human proteomes. Peptides. 2008;29(10):1755-1766.

66. Lucchese G. Understanding neuropsychiatric diseases, analyzing the peptide sharing between infectious agents and the language-associated NMDA 2A Protein. Front Psychiatry. 2016;7:60.

67. Lucchese G. From toxoplasmosis to schizophrenia via NMDA dysfunction: peptide overlap between Toxoplasma gondii and N-Methyld-Aspartate receptors as a potential mechanistic link. Front Psychiatry. 2017;8:37.

68. Lucchese G, Capone G, Kanduc D. Peptide sharing between influenza A H1N1 hemagglutinin and human axon guidance proteins. Schizophr Bull. 2014;40(2):362-375.

69. Lucchese G, Kanduc D. Single amino acid repeats connect viruses to neurodegeneration. Curr Drug Discov Technol. 2014;11(3):214-219.

70. Lucchese G. Confronting JC virus and Homo sapiens biological signatures. Front Biosci. 2013;18:716-724.

71. Lucchese G. A peptide talk between JC virus and the human host: from silent infection to autoimmunity. Immunopharmacol Immunotoxicol. 2012;34(6):1067-1074.

72. Lucchese G, Kanduc D. Minimal immune determinants connect Zika virus, human Cytomegalovirus, and Toxoplasma gondii to microcephalyrelated human proteins. Am J Reprod Immunol. 2017;77(2):e12608.

73. Takahashi T, Suzuki T. Role of sulfatide in normal and pathological cells and tissues. J Lipid Res. 2012;53(8):1437-1450.

74. Ishibashi T, Dupree JL, Ikenaka K, et al. A myelin galactolipid, sulfatide, is essential for maintenance of ion channels on myelinated axon but not essential for initial cluster formation. $J$ Neurosci. 2002;22(15):6507-6514.

75. Piao H, Minohara M, Kawamura N, et al. Tissue binding patterns and in vitro effects of Campylobacter jejuni DNA-binding protein from starved cells. Neurochem Res. 2011;36(1):58-66.

76. Notturno F, Caporale CM, De Lauretis A, Uncini A. Glial fibrillary acidic protein: a marker of axonal Guillain-Barrè syndrome and outcome. Muscle Nerve. 2008;38(1):899-903.

77. Bolis A, Coviello S, Visigalli I, et al. Dlg1, Sec8, and Mtmr2 regulate membrane homeostasis in Schwann cell myelination. J Neurosci. 2009;29(27):8858-8870.

78. Robinson FL, Dixon JE. The phosphoinositide-3-phosphatase MTMR2 associates with MTMR13, a membrane-associated pseudophosphatase also mutated in type 4B Charcot-Marie-Tooth disease. J Biol Chem. 2005;280(36):31699-31707.

79. Nakhro K, Park JM, Hong YB, et al. SET binding factor 1 (SBF1) mutation causes Charcot-Marie-Tooth disease type 4B3. Neurology. 2013;81(2):165-173.

80. Firestein R, Nagy PL, Daly M, Huie P, Conti M, Cleary ML. Male infertility, impaired spermatogenesis, and azoospermia in mice deficient for the pseudophosphatase Sbf1. J Clin Invest. 2002;109(9):1165-1172.
81. Gupta VA, Hnia K, Smith LL, et al. Loss of catalytically inactive lipid phosphatase myotubularin-related protein 12 impairs myotubularin stability and promotes centronuclear myopathy in zebrafish. PLoS Genet. 2013;9(6):e1003583.

82. Bolino A, Bolis A, Previtali SC, et al. Disruption of Mtmr2 produces CMT4B1-like neuropathy with myelin outfolding and impaired spermatogenesis. J Cell Biol. 2004;167(4):711-721.

83. Yin PQ, Sun YY, Chen HP, Li GZ, Zhong D. Genome-wide gene expression analysis of peripheral leukocytes in relation to the male predominance of Guillain-Barre syndrome: differential gene expression between male and female patients. Int J Neurosci. 2016;126(6): 531-541.

84. Takasaki N, Tachibana K, Ogasawara S, et al. A heterozygous mutation of GALNTL5 affects male infertility with impairment of sperm motility. Proc Natl Acad Sci U S A. 2014;111(3):1120-1125.

85. Nagata Y, Yamashiro S, Yodoi J, Lloyd KO, Shiku H, Furukawa K. Expression cloning of beta 1,4 N-acetylgalactosaminyltransferase cDNAs that determine the expression of GM2 and GD2 gangliosides. J Biol Chem. 1992;267(17):12082-12089.

86. Foster AC, Farnsworth J, Lind GE, et al. D-Serine is a substrate for neutral amino acid transporters ASCT1/SLC1A4 and ASCT2/SLC1A5, and is transported by both subtypes in rat hippocampal astrocyte cultures. PLoS One. 2016;11(6): 0156551.

87. Weiss MD, Derazi S, Kilberg MS, Anderson KJ. Ontogeny and localization of the neutral amino acid transporter ASCT1 in rat brain. Brain Res Dev Brain Res. 2001;130(2):183-190.

88. Takei K, Sato M, Nakamura M, Shimizu H. Longitudinally extensive transverse myelitis with anti-NMDA receptor antibodies during a systemic lupus erythematosus flare-up. BMJ Case Rep. 2015.

89. Ishikawa Y, Ikeda K, Murata K, et al. Ophthalmoplegia and flaccid paraplegia in a patient with anti-NMDA receptor encephalitis: a case report and literature review. Intern Med. 2013;52(24): 2811-2815.

90. Long JB, Rigamonti DD, Oleshansky MA, Wingfield CP, MartinezArizala A. Dynorphin A-induced rat spinal cord injury: evidence for excitatory amino acid involvement in a pharmacological model of ischemic spinal cord injury. J Pharmacol Exp Ther. 1994;269(1): 358-366.

91. Wang L, Shao C, Yang C, Kang X, Zhang G. Association of antigangliosides antibodies and anti-CMV antibodies in Guillain-Barré syndrome. Brain Behav. 2017;7(5):e00690.

92. Asthana P, Vong JS, Kumar G, et al. Dissecting the role of antiganglioside antibodies in Guillain-Barré syndrome: an animal model approach. Mol Neurobiol. 2016;53(7):4981-4991.

93. Mansfield KL, Horton DL, Johnson N, et al. Flavivirus-induced antibody cross-reactivity. J Gen Virol. 2011;92(pt 12):2821-2829.

94. Landry ML, St George K. Laboratory diagnosis of Zika virus infection. Arch Pathol Lab Med. 2017;141(1):60-67.

95. Fazekas de St Groth S, Webster RG. Disquisitions of original antigenic sin. I. Evidence in man. J Exp Med. 1966;124(3):331-345.

96. Bardina SV, Bunduc P, Tripathi S, et al. Enhancement of Zika virus pathogenesis by preexisting antiflavivirus immunity. Science. 2017;356(6334):175-180. 


\section{Supplementary materials}

Table SI List of the 97 human proteins related to myelin, (de)myelination, and/or axonal neuropathies and sharing pentapeptides with ZIKV polyprotein (human proteins are reported by UniProt entry, a brief description of function, and aa length)

ABCDI. ATP-binding cassette sub-family D member I. Adrenoleukodystrophy. Progressive multifocal demyelination of the CNS.

ACATN. Acetyl-coenzyme A transporter I. Cerebral and cerebellar atrophy and hypomyelination.

ACY2. Aspartoacylase. White matter vacuolization and demyelination.

ADCY6. Adenylate cyclase type 6. Hypomyelination neuropathy.

ANAG. Alpha- $N$-acetylglucosaminidase. Axonal neuropathies.

ARSA. Arylsulfatase A. Hydrolyzes cerebroside sulfate. Intralysosomal storage of cerebroside-3-sulfate, with a diffuse loss of myelin in the

CNS

CCI77. Myelin proteolipid protein-like protein.

CGT. 2-Hydroxyacylsphingosine I-beta-galactosyltransferase. Synthesis of galactocerebrosides, which are abundant in the myelin of the

CNS and peripheral nervous system.

$\mathrm{CH} 60.60 \mathrm{kDa}$ heat shock protein, mitochondrial precursor. Hypomyelinating leukodystrophy characterized by infantile-onset rotary

nystagmus, progressive spastic paraplegia, neurologic regression, motor impairment, profound mental retardation.

CLCN2. Chloride channel protein 2 (CIC-2). Leukoencephalopathy with ataxia. White matter abnormalities on brain MRI suggesting myelin

CLDI I. Claudin- II. Oligodendrocyte-specific protein. Oligodendrocyte-specific protein is concentrated in CNS myelin, seems to modulate proliferation and migration of oligodendrocytes, is an autoantigen in the development of autoimmune demyelinating disease.

$\mathrm{CMCl}$. Calcium-binding mitochondrial carrier protein Aralarl. Mitochondrial aspartate glutamate carrier I. Epileptic encephalopathy, early infantile, characterized by global cerebral hypomyelination.

CN37. 2',3'-Cyclic-nucleotide 3'-phosphodiesterase. CN37 is the third most abundant protein in CNS myelin.

CNTNI. Contactin-I precursor. Neural cell surface protein F3. Involved in the formation of paranodal axo-glial junctions in myelinated peripheral nerves. Myopathy, hypotonia, muscle weakness.

CNTPI. Contactin-associated protein I. Involved in the saltatory conduction of nerve impulses in myelinated nerve fibers; demarcates the paranodal region of the axo-glial junction; may have a role in the signaling between axons and myelinating glial cells. Axoglial disease characterized by degeneration of anterior horn neurons, extreme skeletal muscle atrophy, and joint contractures leading to various degrees of flexion or extension limitations.

CNTP2. Contactin-associated protein-like 2. Function and pathology as for CNTPI.

CTDPI. RNA polymerase II subunit A C-terminal domain phosphatase. Hypomyelination of the peripheral nervous system.

CTLI. Choline transporter-like protein I. May be involved in myelin production.

CXBI. Gap junction beta-I protein. Connexin-32. Associated with both demyelinating and axonal neuropathies.

CXG2. Gap junction gamma-2 protein. Connexin-46.6. Hypomyelinating leukodystrophy with symptoms of Pelizaeus-Merzbacher

disease.

CXG3. Gap junction gamma-3 protein. Connexin-30.2. Expressed within myelinating glial cells of the CNS and peripheral nervous system.

DNJB2. DNAJ homolog subfamily B member 2. Axonal neuropathies. Muscle weakness and atrophy resulting in gait impairment and loss of reflexes due to impaired function of motor nerves.

DPYL2. Dihydropyrimidinase-related protein 2. Collapsin response mediator protein 2. Neurodegeneration.

DRP2. Dystrophin-related protein 2. Required for normal myelination and the formation of Cajal bands in myelinating Schwann cells.

$\mathrm{DYHCI}$. Cytoplasmic dynein I heavy chain I. Axonal neuropathy. Neuromuscular disorder characterized by degeneration of the anterior horn cells of the spinal cord, leading to symmetrical muscle weakness and atrophy.

EGR2. E3 SUMO-protein ligase EGR2. Hypomyelinating/amyelinating neuropathies.

ENOG. Gamma-enolase. Has neurotrophic and neuroprotective properties on a broad spectrum of CNS neurons.

ENPP6. Ectonucleotide pyrophosphatase/phosphodiesterase family member 6. Choline-specific glycerophospho-diester

phosphodiesterase.

EXOS8. Exosome complex component RRP43, cerebellar and corpus callosum hypoplasia, abnormal myelination of the CNS, and spinal motor neuron disease.

EZRI. Ezrin. Cytovillin. Expressed in cerebral cortex, basal ganglia, hippocampus, hypophysis, and optic nerve.

FI68B. Myelin-associated neurite-outgrowth inhibitor. Modulates neurogenesis. Expressed in the brain, within neuronal axonal fibers, and 
Table SI (Continued)

GFAP. Glial fibrillary acidic protein. Leukodystrophy with macrocephaly, seizures, and psychomotor retardation 432

GNAO. Guanine nucleotide-binding protein G(o) subunit alpha. Epileptic encephalopathy. Brain abnormalities, such as cerebral atrophy or

thin corpus callosum.

GPM6A. Neuronal membrane glycoprotein M6-a. Involved in neuronal differentiation, including differentiation and migration of neuronal

stem cells.

GPR6. G-protein coupled receptor 6 (sphingosine-I-phosphate receptor GPR6). Blocks myelin inhibition in neurons.

HS7IA. Associates with myelin basic protein and proteolipid protein in multiple sclerosis brains.

641

HS90A. Protection of oligodendrocyte precursor cells.

HSPBI. Heat shock protein beta-I. Axonal neuropathy.

HSPB8. Heat shock protein beta-8. Axonal neuropathy.

HTRAI. Serine protease HTRAI. Demyelination of the cerebral white matter with sparing of $U$ fibers

HYCCI. Hyccin. Downregulated by CTNNBI protein A. Leukodystrophy, hypomyelinating.

IL7RA. Interleukin-7 receptor subunit alpha. Multiple sclerosis

732

205

KIFIB. Kinesin-like protein KIFIB (KIP). Axonal neuropathy.

196

480

521

459

LMNA. Prelamin-A/C.b Axonal neuropathy.

LMNBI. Lamin-BI precursor. Axonal neuropathy.

LRSMI. E3 ubiquitin-protein ligase LRSAMI.

586

Axonal neuropathies in the absence of myelin alterations.

MAG. Myelin-associated glycoprotein precursor.

MAL. Myelin and lymphocyte protein.

MERL. Merlin.Moesin-ezrin-radixin-like protein. Neurofibromin-2. Schwannomin.

MI. S-Adenosylmethionine synthase isoform type-I. Brain demyelination due to methionine adenosyltransferase deficiency.

MRF Myelin regulatory factor.

MRFL Myelin regulatory factor-like protein.

MTMR2. Myotubularin-related protein 2. MTMR2. Demyelination.

910

MTMR5. Myotubularin-related protein 5. Demyelination.

MTMRD. Myotubularin-related protein I3. Demyelinating neuropathy.

MYEF2. Myelin expression factor 2.

1849

MYOID. Unconventional myosin-Id. Expressed in myelinating oligodendrocytes.

600

MYPR. Myelin proteolipid protein. It is the major myelin protein from the CNS. Hypomyelinating leukodystrophy.

MYTI. Myelin transcription factor I. May play a role in: development of neurons and oligodendroglia in the CNS; differentiation of

oligodendrocytes; regulation of myelin gene transcription.

MYTIL. Myelin transcription factor I-like protein. May play a role in development of neurons and oligodendroglia in the CNS. II86

NDRGI. Protein NDRGI. Demyelinating neuropathy.

394

NFH. Neurofilament heavy polypeptide. Amyotrophic lateral sclerosis. Axonal degeneration in the absence of myelin alterations.

NFL. Neurofilament light polypeptide. Demyelinating neuropathy.

1026

543

NRCAM. Neuronal cell adhesion molecule precursor. Plays a role in the formation and maintenance of the nodes of Ranvier on

myelinated axons.

OPALI. Opalin. Oligodendrocytic myelin paranodal and inner loop protein.

P5CR2. Pyrroline-5-carboxylate reductase 2. Hypomyelinating leukodystrophy.

PARD3. Partitioning defective 3 homolog, modulates peripheral myelination

PRAX. Periaxin. Demyelinating neuropathy.

PTPRC. Receptor-type tyrosine-protein phosphatase C. Multiple sclerosis.

RPACI. DNA-directed RNA polymerases I and III subunit RPACI. Hypomyelinating leukodystrophy.

RPCI. DNA-directed RNA polymerase III subunit RPCI. Hypomyelinating leukodystrophy. 
Table SI (Continued)

SYDC. Aspartate-tRNA ligase, cytoplasmic. Hypomyelination and white matter lesions in the cerebrum, brainstem, cerebellum, and spinal cord.

SYG. Glycine-tRNA ligase. Axonal neuropathy.

SYHC. Histidine-tRNA ligase, cytoplasmic. Axonal neuropathy.

SYRC. Arginine-tRNA ligase, cytoplasmic. Leukodystrophy. Ataxia associated with diffuse hypomyelination apparent on brain MRI.

TEN4. Teneurin-4. Regulates the myelination of small-diameter axons in the CNS. Essential tremor.

TNRIA. Tumor necrosis factor receptor superfamily member I. Involved in multiple sclerosis.

TRIM2, Tripartite motif-containing protein 2. Axonal neuropathy.

TRPV4. Transient receptor potential cation channel subfamily $V$ member 4. Axonal neuropathy.

871

Notes: Human proteins were retrieved using "myelin, (de)myelination, axonal neuropathy" as keywords. Proteins are indicated by UniProtKB/Swiss-Prot entry names, aa length, and listed in alphabetical order. Details and references for disease involvement are available at http://www.uniprot.org/.

Abbreviations: ZIKV, Zika virus; aa, amino acids; CNS, central nervous system; MRI, magnetic resonance imaging.

Table S2 Pentapeptide platform shared by ZIKV polyprotein and human proteins related to myelin, (de)myelination, and/or axonal neuropathies: 222 ZIKV pentapeptides (in bold) recur throughout 97 proteins (in parentheses as UniProt entry names)

AAAAR (CXG3); AAAIF (GELS); AAARA (NFH); AALGA (GPR6); AARGY (ARSA); AEEVL (SYG); AEMEE (MTMR2); AETDE (TEN4); AGGFA (PRAX); AKFTC (CXG2); AKVEV (NFH); ALAGA (ARSA; CLCN2; GPM6A); ALAGG (DPYL2); ALEAE (CTDPI; SCRIB); ALGAI (NRCAM); ALGLT (ANAG); ALKDG (TEN4); ALRGL (SATT); ALVAV (SYDC); APAYS (FI68B; HSPBI); ARRAL (LMNBI; MYEF2); ASAGI (MRF); ASDSR (ANAG); ASSLV (MAL); AVLLR (MTMR5); CRECT (TRIM2); CSAVP (MYPR); CYSQL (IL7RA); DENHP (MTMR5); DGLSE (FIG4); DHSGK (DRP2); DIEMA (SYDC); DRRWC (TRPV4); DTVNM (CXG3); EALIT (CTLI); EALRG (SCRIB); EDVNL (RPC2); EEARR (ABCDI; EZRI); EEEKE (DYHCI; HS90A; NFH); EEIRR (MFN2); EELEI (MRFL); EEPML (CXG2); EFEAL (EZRI); EFGKA (MYOID); EGLKK (TRIM2); EKEWK (SYRC); ELGKR (FGD4); ENEAL (MRFL); ENIKD (DYHCI); EPARI (SCRIB); ERLQR (SMBP2); ESSSS (MTMRD); ETLGE (SCRIB); ETLHG (NDRGI); EVEET (NFL); EVQLL (PRAX); FATTL (DRP2); FDLEN (ACY2); FPDSN (TRPV4); FVVDG (MYOID); GAALG (CXG3); GAALR (CNTPI; SATT); GAGKT (DYHCI; MYOID); GALEA (ANAG); GCGLF (SMBP2); GDSYI (GELS); GDTAW (SYAC); GEAAA (NFH); GEAGA (PRAX); GERAR (SMBP2); GESSS (GDIA); GETLG (SCRIB); GGGCA (CH60); GGGTG (RTN4R); GIMLL (CTLI); GKRKR (LMNBI); GLDFS (CMCI); GLKKR (MPZL3); GLLIV (MTMR5); GPSLR (MTMR5; HS90A); GQVVT (LMNA; PRAX); GRARV (GELS); GSASS (CCI77; CXG2); GSQHS (IL7RA); GTLPG (RTN4R); GTRGP (SCRIB); GTVSL (PARD3); GVPLL (SMBP2); HFSLG (MFN2); HSDLG (MAG); IAACL (CLCN2); IEPAR (SCRIB); IFLST (DYHCI); IILLV (TRPV4); IKDTV (MERL); ILAAL (SAP); ILAFL (S3TC2); ILLMV (GPM6A); IPGLQ (S3TC2); IPKSL (KIFIB); ISALE (NRCAM); ISRQD (TEN4); KEVKK (EXOS8); KGIGK (RPACI); KGSLV (SYAC); KKSGI (MYTIL); KNPKE (KIFIB); KTKDG (HSPBI; HSPB8); LAAAV (S3TC2); LAGAL (CGT; GFAP; MTMR2); LALGG (P5CR2); LDFSD (CMCI); LEERG (FGD4); LEGDL (LMNBI); LGLQR (MYOID); LIYTV (MTMRD); LKDGR (TEN4); LKDGV (SYHC); LKGKG (CH60); LKMDK (MERL); LLALA (ABCDI; ARSA; ENPP6; S3TC2); LLAVP (ACATN); LLGLL (CLCN2; CNTNI; SATT); LLLGR (HTRAI); LLLLT (CLDII); LLSLK (HYCCI); LLTRS (P5CR2); LLTTA (CH60); LLVVL (PTPRC); LNDMG (NRCAM); LQDGL (MTMR5); LRGLP (CN37); LRIIN (HS7IA); LSTQV (ACATN); LTAVG (CXG2); LTAVR (RTN4R); LTCLA (IL7RA); LVDRE (KIFIB); LVEED (MYTI); LVILL (HS90A; TEN4); LVNGV (SYRC); LWLLR (ABCDI); MAVLV (ADCY6); MLELD (ENOG); MLLSL (ADCY6); MSWFS (ADCY6); NAALG (SDHA); NGVQL (SYRC); NSFLV (DPYL2); NSTHE (NRCAM); PALLV (CXBI; MTMR2; NDRGI); PFAAG (SCRIB); PFGDS (ARSA); PRRLA (P5CR2); PSLGL (PARD3; OPALI); PTQGS (CN37); PVGRL (CXG3); PVILD (SAP); QLLYF (TRPV4); QRGSG (CMCI); RDLRL (CLCN2; NFL); REEGA (MTMR5); RFEEC (MFN2); RGECH (TEN4); RGYIS (CH60); RLAAA (PRAX); RPALL (NRCAM; MTMRD); RPASA (RTN4R); RRALK (METKI); RRDLR (DYHCI); RRLAA (FA2H); RRLLG (MAG); RRWCF (TRPV4); RVILA (RPC2); SAVPV (MYPR); SEELE (MTMR5); SGKRS (MTMR2); SLFGG (CLCN2; GELS); SLGLD (ADCY6); SLGLI (FIG4; MTMR5); SLRST (DRP2); SLTCL (IL7RA); SPGAG (DNJB2); SSSPE (DYHCI); SSTSQ (HYCCI); STSQK (CNTP2); STTAS (EGR2); TAAGI (P5CR2); TAVSA (STXBI); TEVEV (MAG); TKEEF (CMCI); TKNGS (SMBP2); TLETI (TEN4); TPVGR (CXG3); TQGSA (ARSA); TVDIE (RPC2); TVEVQ (TNRIA); TVSLG (GPR6); VATGG (SDHA); VDGDT (CXG3; MYOID); VEEDG (DNJB2); VEFKD (MYEF2); VEGLG (NRCAM); VFIYN (KIFIB); VLDLH (LRSMI); VLSMV (MTMR5); VLTAV (CXG2); VNPLG (PTPRC); VPERA (RTN4R); VQLLA (CMCI); VRAAK (NFL); VSRGS (TRPV4); VSRME (GNAO); VSYVV (ADCY6); VTLGA (PRAX); VVAAE (CXBI; GPR6); VVDGD (MYOID); VVDPI (RPCI); VVGLL (GDAPI); YISTR (STXBI); YLSTQ (ACATN); YSLMA (ANAG)

Abbreviation: ZIKV, Zika virus.

Virus Adaptation and Treatment

\section{Publish your work in this journal}

Virus Adaptation and Treatment is an international, peer-reviewed open access journal focusing on the study of virology, viral adaptation and the development and use of antiviral drugs and vaccines to achieve improved outcomes in infection control and treatment. The journal welcomes original research, basic science, clinical \& epidemiological studies,
Dovepress

reviews \& evaluations, expert opinion and commentary, case reports and extended reports. The manuscript management system is completely online and includes a very quick and fair peer-review system, which is all easy to use. Visit http://www.dovepress.com/testimonials.php to read real quotes from published authors. 